\title{
Distribution of five common subtypes of spinocerebellar ataxia in the Korean population
}

\author{
In-Hee Choi', Gu-Hwan Kim', Beom-Hee Lee ${ }^{1,2}$, Jin-Ho Choi ${ }^{2}$, and Han-Wook Yoo ${ }^{1,2, *}$ \\ ${ }^{1}$ Medical Genetics Center, Asan Medical Center, Seoul, Korea \\ ${ }^{2}$ Department of Pediatrics, Asan Medical Center Children's Hospital, University of Ulsan College of Medicine, Seoul, Korea
}

Purpose: Spinocerebellar ataxia (SCA) is a genetically heterogeneous disease for which more than 30 subtypes have been identified. However, 5 subtypes, SCA1, SCA2, SCA3, SCA6, and SCA7, account for more than $60 \%$ of cases. In this study, we report the distribution of these 5 subtypes in Korean patients.

Materials and Methods: Six hundred and thirty-eight unrelated patients with a presumptive diagnosis of SCA were included in this study. Trinucleotide (CAG) repeat number (TNR) repeat number was determined using fluorescently labeled primers and fragment analysis.

Results: A total of 128 unrelated patients (20.1\% of all individuals tested) tested positive for SCA subtypes, including SCA1 (5 patients, 3.9\% of those testing positive), SCA2 (38 patients, 29.7\%), SCA3 (30 patients, 23.4\%), SCA6 (39 patients, 30.5\%), and SCA7 (16 patients, $12.5 \%$ ). The mean copy number of pathogenic TNR alleles was $45 \pm 8.5$ for SCA1, $42 \pm 3.1$ for SCA2, 72 \pm 5.4 for SCA3, 23 \pm 1.5 for SCA6, and 50 \pm 11.4 for SCA7. TNR copy number was inversely correlated with onset age in SCA2, SCA6, and SCA7.

Conclusion: SCA2, SCA3, and SCA6 are common SCA subtypes in Korean patients and could be screened as a first-line test. Expanded pathogenic allele size was associated with early onset age.

Key words: Spinocerebellar ataxias, Trinucleotide repeats.

\section{Introduction}

Spinocerebellar ataxia (SCA) consists of a group of disorders that are clinically and genetically heterogeneous. SCA is characterized by progressive ataxia of gait, stance, and limbs. In addition, cerebellar dysarthria, oculomotor disturbances of cerebellar and supranuclear origin, retinopathy, optic atrophy, spasticity, extra-pyramidal signs, peripheral neuropathy, sphincter disturbances, cognitive impairment, and epilepsy are noted in varying degrees of severity $[1,2]$

To date, more than 30 SCAs have been identified. However, it is very difficult to diagnose a particular subtype of SCA on a clinical basis because of phenotypic overlap among these subtypes [1]. Genetic diagnosis has also proved to be challenging, as more than 30 different loci have been implicated so far [1]. However, epidemiological data have revealed that 5 autosomal dominant subtypes, SCA1, SCA2, SCA3, SCA6, and SCA7, caused by abnormal trinucleotide (CAG) repeat expansion, account for more than $60 \%$ of all SCAs [3]. The expanded CAG-

Received: 8 December 2014, Revised: 10 December 2014, Accepted: 10 December 2014, Published: 31 December 2014

*Corresponding author: Han-Wook Yoo, M.D., Ph.D.

Medical Genetics Center, Asan Medical Center and Department of Pediatrics, Asan Medical Center Children's Hospital, University of Ulsan College of Medicine, 88 Olympic-ro 43-gil, Songpa-gu, Seoul 138-736, Korea.

Tel: +82-2-3010-3374, Fax: +82-2-473-3725, E-mail: hwyoo@amc.seoul.kr

Conflict of interest: We declare that we do not have any conflicts of interests.

(c) This is an open-access article distributed under the terms of the Creative Commons Attribution Non-Commercial License (http://creativecommons.org/licenses/by-nc/3.0/) which permits unrestricted non-commercial use, distribution, and reproduction in any medium, provided the original work is properly cited.

(c) Copyright 2014 by the Korean Society of Medical Genetics 
Table 1. SCA subtype PCR primer sequences and ranges of trinucleotide copy numbers and PCR product sizes

\begin{tabular}{|c|c|c|c|c|c|c|c|c|c|c|}
\hline Type & & Primer sequences & $\begin{array}{l}\text { Annealing } \\
\text { temp. }\left({ }^{\circ} \mathrm{C}\right)\end{array}$ & $\begin{array}{l}\text { DMSO } \\
(\%)\end{array}$ & Gene & Location & RefSeq ID & & $\begin{array}{l}\text { CAG } \\
\text { repeat } \\
\text { number }\end{array}$ & $\begin{array}{c}\text { PCR } \\
\text { product size } \\
\text { (bp) }\end{array}$ \\
\hline SCA1 & $\begin{array}{l}1 \text { Sense } \\
\text { Antisense }\end{array}$ & $\begin{array}{l}\text { GGGCCACCACTCCATCCCAG } \\
\text { GCGGAGAACTGGAAATGTGGAC }\end{array}$ & 58 & 7.5 & ATXN1 & 6p23 & NT_007592.15 & $\begin{array}{l}\text { Healthy } \\
\text { Pathogenic }\end{array}$ & $\begin{array}{l}6-39 \\
41-83\end{array}$ & $\begin{array}{l}204-303 \\
309-435\end{array}$ \\
\hline SCA2 & $\begin{array}{l}2 \text { Sense } \\
\text { Antisense }\end{array}$ & $\begin{array}{l}\text { GGGCCCCTCACCATGTCG } \\
\text { CGGGCTTGCGGACATTGG }\end{array}$ & 63 & 2.5 & ATXN2 & $12 \mathrm{q} 24.1$ & NT_009775.17 & $\begin{array}{l}\text { Healthy } \\
\text { Pathogenic }\end{array}$ & $\begin{array}{l}14-32 \\
34-77\end{array}$ & $\begin{array}{l}103-157 \\
163-292\end{array}$ \\
\hline SCA3 & $\begin{array}{l}3 \text { Sense } \\
\text { Antisense }\end{array}$ & $\begin{array}{l}\text { CCAGTGACTACTTTGATTCG } \\
\text { GTAACCTTGCTCCTTAATCC }\end{array}$ & 58 & 0 & ATXN3 & $14 \mathrm{q} 32.1$ & NT_026437.12 & $\begin{array}{l}\text { Healthy } \\
\text { Pathogenic }\end{array}$ & $\begin{array}{l}12-40 \\
62-86\end{array}$ & $\begin{array}{l}400-484 \\
550-622\end{array}$ \\
\hline SCA6 & $\begin{array}{l}6 \text { Sense } \\
\text { Antisense }\end{array}$ & $\begin{array}{l}\text { CACGTGTCCTATTCCCCTGTGATCC } \\
\text { TGGGTACCTCCGAGGGCCGCTGGTG }\end{array}$ & 68 & 2.5 & CACNA1A & 19p13 & NT_011295.11 & $\begin{array}{l}\text { Healthy } \\
\text { Pathogenic }\end{array}$ & $\begin{array}{c}7-18 \\
21-27\end{array}$ & $\begin{array}{l}123-156 \\
165-183\end{array}$ \\
\hline SCA7 & $\begin{array}{l}7 \text { Sense } \\
\text { Antisense }\end{array}$ & $\begin{array}{l}\text { TAGGAGCGGAAAGAATGTCG } \\
\text { AGCCTCAACCCACAGATTCC }\end{array}$ & 55 & 7.5 & ATXN7 & 3p21.2-p12 & NT_022517.18 & $\begin{array}{l}\text { Healthy } \\
\text { Pathogenic }\end{array}$ & $\begin{array}{c}7-18 \\
38-200\end{array}$ & $\begin{array}{l}308-341 \\
401-887\end{array}$ \\
\hline
\end{tabular}

SCA, spinocerebellar ataxia; PCR, polymerase chain reaction; temp., temperature; DMSO, dimethyl sulfoxide, RefSeq ID, reference sequence identifier.

repeat sequence encodes toxic polyglutamine tracts in peptides, leading to an accumulation of misfolded mutant proteins. An increase in the number of repeats over a certain level is associated with the manifestation of this neurodegenerative disease, and age at onset is known to be inversely correlated with repeatexpansion size [4].

Autosomal dominant cerebellar ataxias are estimated to occur in 1 to 5 individuals per 100,000 in global population [4]. The distribution of SCA subtypes has been described in many different populations. Although SCA3 predominates worldwide, the most common subtype differs in each population: SCA1 in South Africa, SCA2 in India and Singapore, SCA3 in Europe and China, and SCA6 in Japan [1,5-11].

The distribution of some SCA subtypes in Korea has been reported in a small number of studies. However, the number of patients included was too small to estimate the overall distribution of SCA in the Korean population $[12,13]$. In addition, genetic tests were carried out in multiple centers using different methods, limiting the accuracy of the data obtained [14]. Therefore, this study analyzed genetic data using a single method, and in order to provide more informative data allowing accurate estimation of the distribution in the Korean population of the 5 most common SCAs, we enrolled a large number of Korean patients for whom genetic testing for SCA had been carried out in Medical Genetics Center, Asan Medical Center.

\section{Materials and Methods}

The Institutional Review Board of Asan Medical Center, Seoul, Korea, approved the current study (\#2011-220). This study included 638 unrelated Korean patients for whom genetic testing of SCA had been carried out at the Medical Genetics Center, Asan Medical Center from 2000 to 2009. Patients presumptively diagnosed as having SCA had at least one of the following major manifestations of the disease: cerebral atrophy, progressive ataxia, gait disturbance, dysarthria, and/ or parkinsonism. Age at the time of testing ranged from 9 to 74 years (mean \pm standard deviaton [SD]: $45 \pm 15$ years). Polymerase chain reaction (PCR) was performed with Taq polymerase (GoTaq; Promega, Madison, WI, USA), and specific primers for SCA1, SCA2, SCA3, SCA6, and SCA7 were designed using Primer3 (http://www.bioinformatics.nl/cgi-bin/primer3plus/ primer3plus.cgi/). The primer sequences and their reference accession numbers are listed in Table 1. The 5 '-ends of forward primers were labeled with 6-fluorescein amidite, and PCR products were analyzed using an ABI3130xI Genetic Analyzer (Applied Biosystems, Foster City, CA, USA). PCR product sizes were measured using the GeneScan LIZ500 marker (Applied Biosystems), and CAG repeat numbers were calculated using PCR product sizes and corresponding reference sequences (Table 1). Parameters are presented as ranges.

Statistical analyses were carried out using SPSS software for Windows, ver. 12.0 (SPSS Inc., Chicago, IL, USA). Correlations between $C A G$ repeat number and age at request for genetic testing were analyzed using Pearson's correlation coefficient. A $P<0.05$ was considered to be statistically significant.

\section{Results}

Out of 638 unrelated patients, 128 (20.1\%) tested positive 
for SCA. SCA 1 was diagnosed in 5 patients (3.9\%), SCA2 in 38 patients (29.7\%), SCA3 in 30 patients (23.4\%), SCA6 in 39 patients (30.5\%), and SCA7 in 16 patients (12.5\%; Fig. 1). SCA2, SCA3, and SCA6 were the most prevalent subtypes.

The healthy $C A G$ repeat number ranges of genes implicated in SCA1, SCA2, SCA3, SCA6, and SCA7 were 6-39 in ATXN1, 1432 in ATXN2, 12-40 in ATXN3, 7-18 in CACNA1A, and 7-18 in ATXN7, respectively. The mean number of CAG repeats in pathogenic alleles was $45.4 \pm 8.5$ (range, 38-60) in ATXN1 in patients with SCA1, 42.3 \pm 3.1 (36-50) in ATXN2 in patients with SCA2, $72.1 \pm 5.4$ (54-80) in ATXN3 in patients with SCA3, 23.0 $\pm 1.5(21-$ 27) in CACNA1A in patients with SCA6, and 50.1 $\pm 11.4(42-80)$ in ATXN7 in patients with SCA7.

The male-to-female ratio was $3: 2$ for SCA1, $17: 21$ for SCA2, $13: 17$ for SCA3, $25: 14$ for SCA6, and $5: 11$ for SCA7. In all, 65 patients were women and 63 were men (Table 2).

Number of CAG repeats was inversely correlated with age at

SCA2

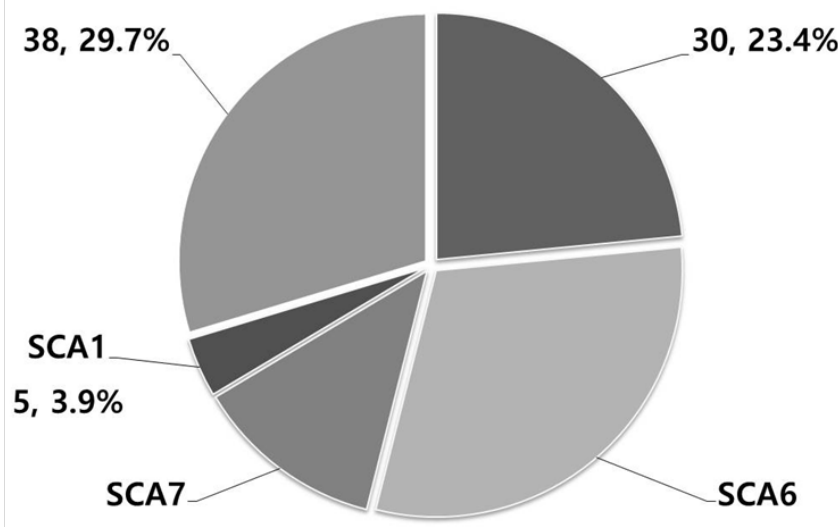

$16,12.5 \%$

$39,30.5 \%$

Fig. 1. Frequencies of spinocerebellar ataxia (SCA) subtypes. SCA subtypes were determined by fragment analysis using polymerase chain reaction (PCR) and capillary electrophoresis. The numbers of patients were 5 for SCA1, 38 for SCA2, 30 for SCA3, 39 for SCA6, and 16 for SCA7. testing for SCA2 $(r=-0.416, P=0.011)$, SCA6 $(r=-0.575, P=0.001)$, and SCA7 ( $r=-0.696, P=0.043)$. However, this inverse correlation was not significant for SCA1 and SCA3 (Fig. 2).

\section{Discussion}

The frequencies of SCA subtypes have been reported in many other ethnic groups, and have been found to differ among them [1-2]. However, the most common SCA subtypes throughout these studies have consistently been SCA1, SCA2, SCA3, SCA6, and SCA7. The current study described the frequencies of these 5 common SCA subtypes in 128 Korean patients at a single center.

Using data from one of the biggest centers performing genetic testing for SCA in Korea, our report indicates a few points comparable to those of previous studies. Recently, Kim et al. [14] reported the distribution of SCAs from multiple centers in Korea, constituting the largest study of SCA in the Korean population reported to date. They examined 351 cases of SCA1, SCA2, SCA3, SCA6, SCA7, SCA8, SCA17, SCA31, and dentatorubropallidoluysian atrophy. In their report, SCA2 (28\%), SCA3 (22\%), and SCA6 (23\%), were common subtypes, with SCA2 being the most common, a finding different from those in other East Asian countries including China and Japan where SCA 3 and SCA6 are commonly found, but SCA2 is not. In line with this observation, SCA2, SCA3, and SCA6 were the most frequently observed subtypes in our study. However, our data showed SCA6 to be the most prevalent. Nevertheless, it is a notable finding that SCA2 was common subtype in our data that has also been identified as a common subtype in the Korean population in other studies $[13,14]$.

Some interactions have been observed between age at onset and expanded CAG repeat number. Because the number of

Table 2. Summary of patient characteristics

\begin{tabular}{|c|c|c|c|c|c|c|c|}
\hline Variable & SCA1 & SCA2 & SCA3 & SCA6 & SCA7 & Average & Total \\
\hline Patient & $5(3.9)$ & $38(29.7)$ & $30(23.4)$ & $39(30.5)$ & $16(12.5)$ & & $128(100)$ \\
\hline Gender (male : female) & $\begin{array}{c}3: 2 \\
(1: 0.7)\end{array}$ & $\begin{array}{c}17: 21 \\
(1: 1.2)\end{array}$ & $\begin{array}{c}13: 17 \\
(1: 0.8)\end{array}$ & $\begin{array}{l}25: 14 \\
(1: 0.6)\end{array}$ & $\begin{array}{c}5: 11 \\
(1: 2.2)\end{array}$ & & $\begin{array}{c}63: 65 \\
(1: 1)\end{array}$ \\
\hline Age at request (yr) & $\begin{array}{c}39.2 \pm 12.0 \\
(27-54)\end{array}$ & $\begin{array}{c}40.4 \pm 11.6 \\
(20-65)\end{array}$ & $\begin{array}{c}45.0 \pm 11.1 \\
(19-65)\end{array}$ & $\begin{array}{c}55.8 \pm 10.3 \\
(36-74)\end{array}$ & $\begin{array}{c}40.6 \pm 11.5 \\
(9-67)\end{array}$ & $\begin{array}{c}46.2 \pm 13.3 \\
(9-74)\end{array}$ & \\
\hline CAG repeat numbers & $\begin{array}{c}45.4 \pm 8.5 \\
(38-60)\end{array}$ & $\begin{array}{c}42.3 \pm 3.1 \\
(36-50)\end{array}$ & $\begin{array}{c}72.1 \pm 5.4 \\
(54-80)\end{array}$ & $\begin{array}{c}23.0 \pm 1.5 \\
(21-27)\end{array}$ & $\begin{array}{c}50.1 \pm 11.4 \\
(42-80)\end{array}$ & & \\
\hline
\end{tabular}

Values are presented as number (\%), or mean \pm standard deviation (range).

SCA, spinocerebellar ataxia. 
A

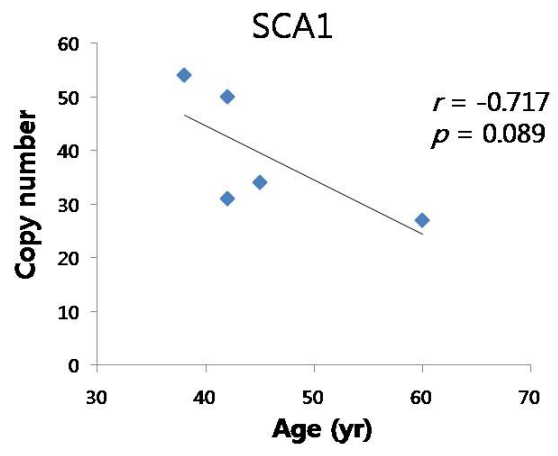

D

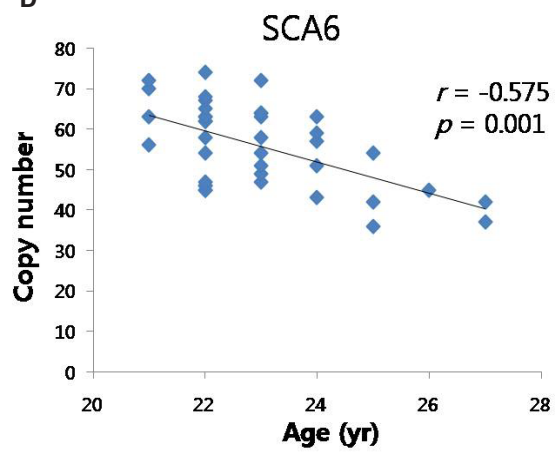

B

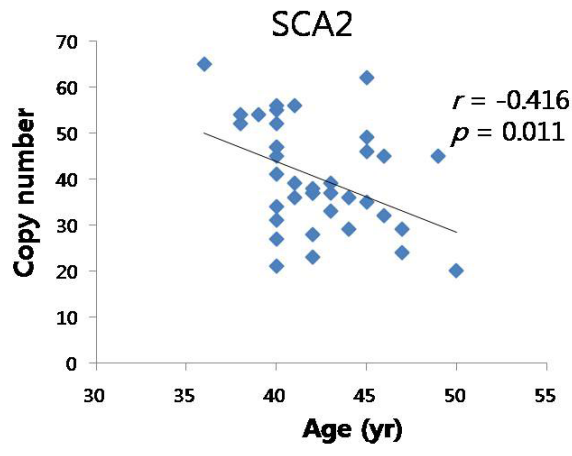

E

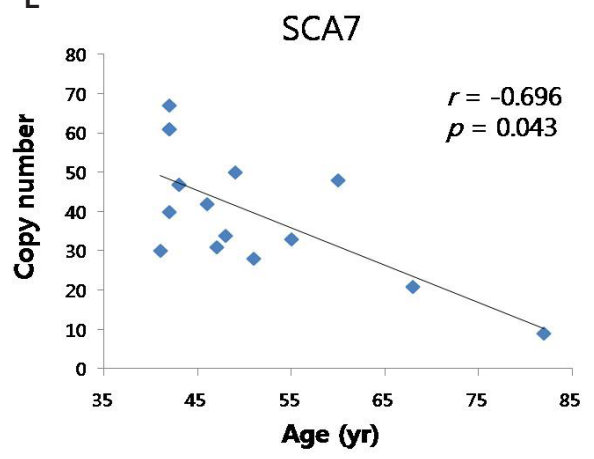

C

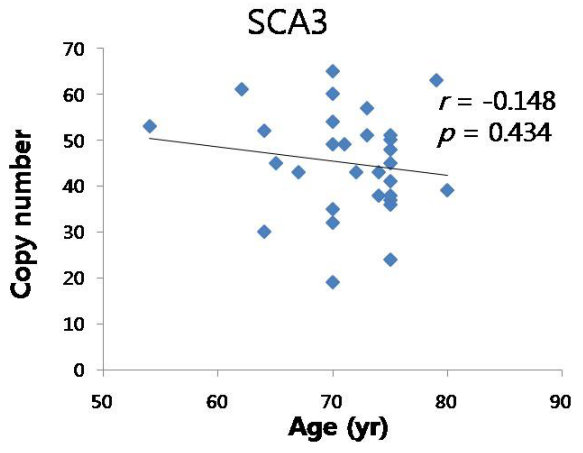

Fig. 2. Correlations between age at request for genetic testing and copy number of CAG repeats in SCA1 (A), SCA2 (B), SCA3 (C), SCA6 (D), and SCA7 (E). SCA, spinocerebellar ataxia.

repeats can be expanded by intergenerational inheritance, known as anticipation, onset age can be young in some patients with large CAG repeat numbers. In our study, the overall inverse correlation between age and repeat number was also observed for all SCA subtypes, although it was only statistically significant for SCA2, SCA6, and SCA7. The small sample size of the current study might explain this discrepancy between SCA subtypes.

Fragment analysis with fluorescently labeled primers is a widely used method for CAG repeat number determination. Determining exact copy number using PCR amplification can be problematic because of repetitive sequences. For this study, we developed optimized PCR conditions with newly designed primer sets that were selected following several trials of various primer pairs. The optimized conditions finally yielded precise repeat numbers, enabling classification of expanded alleles as pathogenic. In addition, information regarding exact copy number is important for health care providers when counseling families affected by SCA regarding intergenerational anticipation, in that copy number expansion is related to the early onset and severity of this disease.

Aside from it being a single-center study, there are other limitations to our current work that are worth noting. Because only age at request, not onset age, was available when genetic testing was carried out for each subject, the median age of patients who were genetically diagnosed as having one of the SCA subtypes does not represent the actual age at clinical presentation and/or diagnosis. In addition, because the patients enrolled in the current study had presumptive diagnoses of SCA, the positive rate of $20.1 \%$ (including all 5 subtypes) does not constitute the true positive rate, which has been reported as being over $60 \%$ in previous studies [3]. The low positive rate might derive from loose clinical diagnostic criteria for genetic testing.

Nevertheless, our study provides further information supporting the observation that 3 SCAs (SCA2, SCA3, and SCA6) are common types in Korea, SCA6 in particular. These 3 subtypes could be screened as a first-line test for genetic diagnosis of SCA in Korean patients. Based on the single methodological approach, our study provided precise CAG repeat numbers, which represents valuable information for clinical practice as well as statistical analysis. Considering the wide range of genetic heterogeneities - more than 30 loci are involved in the SCA disease group - further study is required to develop more commercially available methods able to screen multiple genetic loci in order to enhance the rate of genetic diagnosis. 


\section{Acknowledgements}

This research was supported by a grant from the National Research Foundation of Korea and funded by the Ministry of Education, Science, and Technology (grant No. 2011-0019674).

\section{References}

1. Bird TD. Hereditary Ataxia Overview. [http://www.ncbi.nlm.nih.gov/ books/NBK1138/]

2. Schöls L, Bauer P, Schmidt T, Schulte T, Riess O. Autosomal dominant cerebellar ataxias: clinical features, genetics, and pathogenesis. Lancet Neurol 2004;3:291-304.

3. Durr A. Autosomal dominant cerebellar ataxias: polyglutamine expansions and beyond. Lancet Neurol 2010;9:885-94.

4. Orr HT, Zoghbi HY. Trinucleotide repeat disorders. Annu Rev Neurosci 2007;30:575-621.

5. Ruano L, Melo C, Silva MC, Coutinho P. The global epidemiology of hereditary ataxia and spastic paraplegia: a systematic review of prevalence studies. Neuroepidemiology 2014;42:174-83.

6. Moseley ML, Benzow KA, Schut $\amalg$, Bird TD, Gomez CM, Barkhaus PE, et al. Incidence of dominant spinocerebellar and Friedreich triplet repeats among 361 ataxia families. Neurology 1998;51:1666-71.

7. Saleem Q, Choudhry S, Mukerji M, Bashyam L, Padma MV,

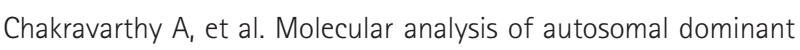
hereditary ataxias in the Indian population: high frequency of
SCA2 and evidence for a common founder mutation. Hum Genet 2000;106:179-87.

8. Storey E, du Sart D, Shaw JH, Lorentzos P, Kelly L, McKinley Gardner $\mathrm{RJ}$, et al. Frequency of spinocerebellar ataxia types 1, 2, 3, 6, and 7 in Australian patients with spinocerebellar ataxia. Am J Med Genet 2000;95:351-7.

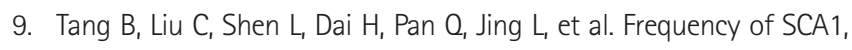
SCA2, SCA3/MJD, SCA6, SCA7, and DRPLA CAG trinucleotide repeat expansion in patients with hereditary spinocerebellar ataxia from Chinese kindreds. Arch Neurol 2000;57:540-4.

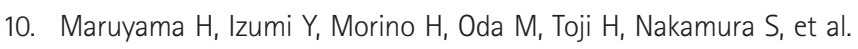
Difference in disease-free survival curve and regional distribution according to subtype of spinocerebellar ataxia: a study of 1,286 Japanese patients. Am J Med Genet 2002;114:578-83.

11. Brusco A, Gellera C, Cagnoli C, Saluto A, Castucci A, Michielotto C, et al. Molecular genetics of hereditary spinocerebellar ataxia: mutation analysis of spinocerebellar ataxia genes and CAG/CTG repeat expansion detection in 225 Italian families. Arch Neurol 2004;61:72733.

12. Lee SG, Ki CS, Kim JW, Suh JS. Distribution of alleles and clinical manifestation in patients with progressive ataxia caused by trinucleotide repeat expansion. Korean J Lab Med 2003;23:60-6.

13. Lee WY, Jin DK, Oh MR, Lee JE, Song SM, Lee EA, et al. Frequency analysis and clinical characterization of spinocerebellar ataxia types 1 , 2, 3, 6, and 7 in Korean patients. Arch Neurol 2003;60:858-63.

14. Kim HJ, Jeon BS, Lee WY, Chung SJ, Yong SW, Kang JH, et al. SCA in Korea and its regional distribution: a multicenter analysis. Parkinsonism Relat Disord 2011;17:72-5. 